\title{
Correlation Histograms in Conductance Measurements of Nanowires Formed at Semiconductor Interfaces
}

\author{
M. Wawrzyniak ${ }^{a}$, J. MartineK $^{b}$, B. SusŁa ${ }^{c}$ And G. Ilnicki ${ }^{b}$ \\ ${ }^{a}$ Faculty of Electronics and Telecommunications, Poznań University of Technology \\ Piotrowo 3A, 60-965 Poznań, Poland \\ ${ }^{b}$ Institute of Molecular Physics, Polish Academy of Sciences \\ M. Smoluchowskiego 17, 60-179 Poznań, Poland \\ ${ }^{c}$ Institute of Physics, Poznań University of Technology \\ Nieszawska 13A, 60-965 Poznań, Poland
}

\begin{abstract}
We demonstrate experimentally that conductance steps can occur in nanowires formed at metal-semiconductor junctions, between a cobalt tip and a germanium surface revealing long-duration plateaus at reproducible levels. The high reproducibility of the conductance traces obtained leads to very sharp peaks in the conductance histogram suggesting formation of stable atomic configurations. We develop a new type of correlation analysis of the preferred conductance values that provide new type of information on a few-atomic-nanocontact formation dynamics.
\end{abstract}

PACS numbers: 68.65.-k, 79.60.Jv, 73.63.Rt

\section{Introduction}

The interesting quantum effects occurring in nanowires with quantum point contact (QPC) can be of use in novel electronic circuits of nanometer dimensions. A QPC occurs when the nanowire width at its neck is comparable to the Fermi wavelength $\lambda_{\mathrm{F}}$. The value of the Fermi wavelength in metals, $\lambda_{\mathrm{F}} \approx 0.5 \mathrm{~nm}$, is comparable to the diameter of a single atom. If, moreover, all the nanowire dimensions are below the electron mean free path $l_{\mathrm{e}}$, the nanowire conductance can be calculated from the Landauer formula $G=G_{0} \sum_{n=1}^{N} T_{n}$, where $G_{0}=2 e^{2} / h$ is the conductance quantum and $T_{n}$ denotes the transmission coefficient. In this range of QPC width and nanowire dimensions the electrical conductance results from $N$ independent electron wave modes, referred to as conductance channels, contributing to the electron transport. When $T_{n}=1$ in each conductance channel, the conductance of a nanowire with $\mathrm{QPC}$ is $G=N G_{0}$, where $N$ denotes the total number of open conductance channels. However, due to hybridization between different bands, or on few-atom level, between different orbitals usually $T_{n}<1$. Its value strongly depends on particular atomic arrangement in a QPC. Typically fabricated by means of a scanning tunneling microscope (STM) [1] and a mechanically controllable break junction (MCBJ) [2], nanowires with QPC have also been produced in relays [3] and between macroscopic wires [4]. In each case, the QPC is achieved by drawing a previously formed nanowire. Obtained while the nanowire is being drawn, conductance versus time plots (conductance traces), reveal steps, which result from the closing of consecutive conductance channels as the nanowire narrows down. As the process of nanowire drawing is by its nature not fully reproducible, conductance histograms are constructed from a large number of conductance traces. In case of gold $[3,4]$ histogram peaks tend to occur around values at integer values of $G_{0}$. The conductance histogram maxima result also from a correlation between the preferred conductance value and the preferred atom arrangement in a single-atom QPC [5]. The method of mechanical fabrication of nanowires has been used for conductance measurements of nanoscale metal-semiconductor contacts $[6,7]$ and semiconductor contacts [8].

This paper reports that conductance steps due to quantum effects can occur in nanowires formed also at metal-semiconductor junctions. The electronic properties of interfaces result from the band structures of the materials in contact, where one can expect the formation of a Schottky barrier.

\section{Experiment}

Our experiment was performed with the $p$-type polycrystalline germanium sample with resistivity of about $0.5 \Omega \mathrm{cm}$ at room temperature and tips made of gold (of purity $99.999 \%$ ) and cobalt (of purity 99.9975\%). The germanium sample was cleaned by grinding the germanium surface. Next, surface regions with suitable cleanness were searched for. Precision piezoelectric actuator 
was used for tip positioning above the germanium surface. The whole mechanical setup was placed on an antivibration table. Nanowires were fabricated by indenting the tip in the surface and retracting it with a nanowire drawn, while the conductance traces were obtained. The measurements were made with a nanowire bias voltage of $400 \mathrm{mV}$ under ambient conditions. Conductance histograms were built from the conductance traces with correction of the analog-to-digital converter differential nonlinearity error $[3,9]$.

\section{Results and discussion}

We record conductance traces for nanowires formed between a gold tip and a gold surface, and between a gold tip and a germanium surface presented in Fig. 1a and $1 b$, respectively. In both cases the tip was brought towards the surface and then retracted at speed $16 \mu \mathrm{m} / \mathrm{s}$; its range of movement in the $z$ direction was $16 \mu \mathrm{m}$. The duration of conductance plateaus in nanowires formed in the gold-germanium system is found to be approximately two orders of magnitude longer than in the goldgold system, despite the same value of retraction speed. This effect can also be seen in other metals and at other retraction speed values. The observed effect is due to complex interplay of unique mechanical and electronic properties of the metal-semiconductors interface. The Fermi energy in germanium, $E_{\mathrm{F}}(\mathrm{Ge}) \approx 0.34 \mathrm{eV}$, is much smaller than in gold, $E_{\mathrm{F}}(\mathrm{Au}) \approx 5.5 \mathrm{eV}$. However, once the $\mathrm{Au}$ tip is indented in the Ge surface, the Fermi level $E_{\mathrm{F}}(\mathrm{Au}-\mathrm{Ge})$ of the metal-semiconductor system shifts to an intermediate value. The corresponding Fermi wavelength values in $\mathrm{Au}$ and $\mathrm{Ge}$ are $\lambda_{\mathrm{F}}(\mathrm{Au}) \approx 0.52 \mathrm{~nm}$ and $\lambda_{\mathrm{F}}(\mathrm{Ge}) \approx 2.1 \mathrm{~nm}$, respectively. Moreover, the mean free path $l_{\mathrm{e}}$ will be longer than in metal due to the lower carrier density at the interface. Also mechanical properties of the nanowires formed between the flat clear crystalline germanium surface and amorphous metal define proper conditions for the formation of stable contacts. Further studies are required to elucidate the mechanism of attaining stable atomic arrangements at semiconductor interface.

Figure 2a shows conductance traces of nanowires with QPC formed at the interface between a Co tip and a Ge surface (traces 1 to 250, step 20). The conductance histogram built from all 250 conductance traces is shown in Fig. 2b. Three long-duration plateaus of reproducible levels can be found in conductance traces, which proves that the process of nanowire forming and drawing was highly reproducible. These three stable conductance plateaus stem from the attainment of stable atomic arrangement. It should be also noted that the range of tip movement in the $z$ direction was $8 \mu \mathrm{m}$, which implies breaking of each nanowire formed. The high reproducibility of the conductance traces leads to very sharp peaks in the conductance histogram. They do not correspond to integer numbers of the quantum conductance indicating a formation of stable atom arrangements. The
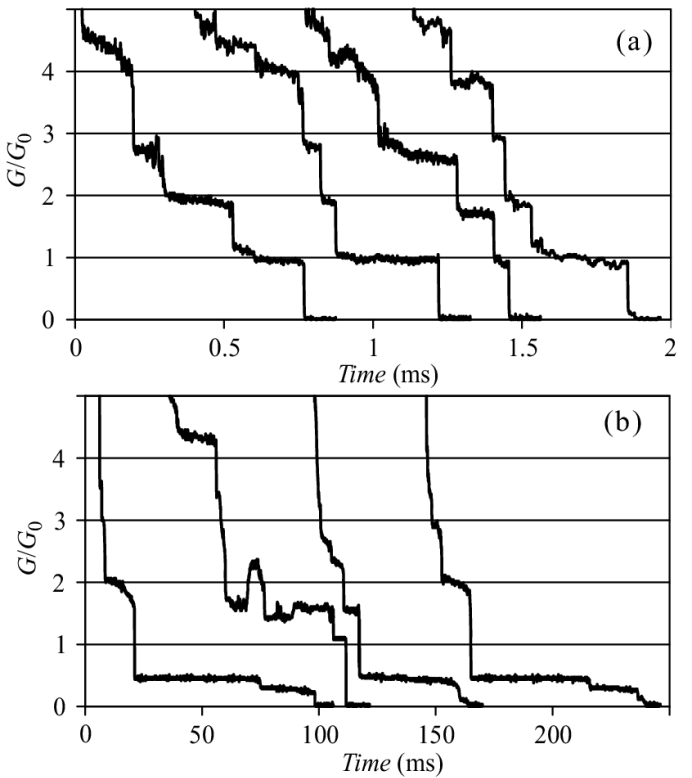

Fig. 1. Conductance traces of nanowires formed between a gold tip and a gold surface (a) and between a gold tip and a germanium surface (b). Measurements were performed under ambient conditions with the tip brought towards the surface, and then retracted at speed $16 \mu \mathrm{m} / \mathrm{s}$ in both cases.
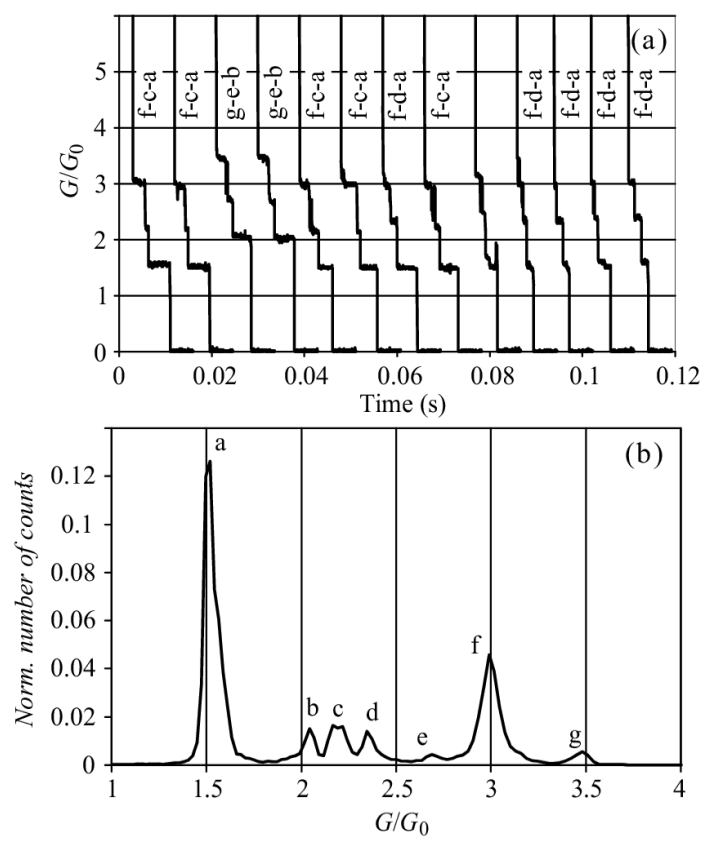

Fig. 2. Conductance measured in nanowires with QPC formed at the interface between a Ge surface and a Co tip: (a) conductance traces 1 to 250 , step 20; we denote by letters a sequence of plateaus that appears in a trace (b) conductance histogram built from all the conductance traces (1 to 250 ) with peaks marked by letters. 


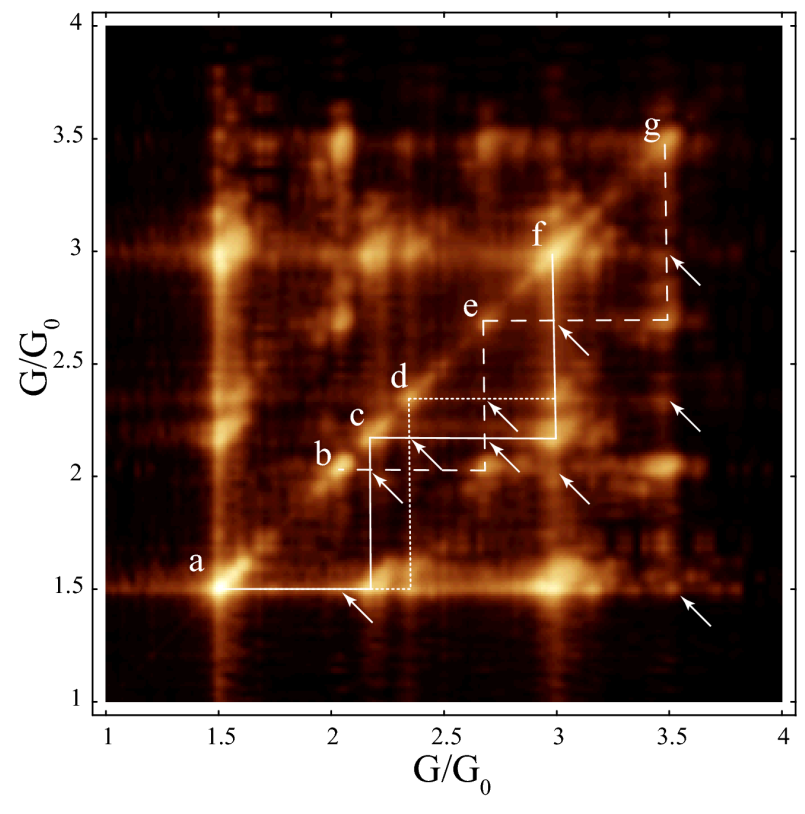

Fig. 3. Conductance correlation histogram in the form of density plot for the same data as in Fig. 2b. The values at the diagonal correspond exactly to plot from Fig. 2b, where corresponding peaks are denoted by letters. Amplitudes of the off-diagonal peaks give the magnitude of the correlation between two different values of the conductance steps. The solid, dotted, and dashed lines correspond to the three typical scenarios of nanowire breaking. White arrows indicate lack of correlation.

conductance then depends on the exact atomic and crystallographic structure of the apexes on both sides of a nanocontact, as well as on the exact electronic structure of the whole nanocontact. Moreover, even assuming the Co nanocontact is of single-atomic size, one still can have more transport channels as both $4 s$ and $3 d$ electrons can contribute to transport ( $2 s$ and $7 d$ electrons in Co). From the conductance traces presented in Fig. 2a, it is possible to find out that there are much fewer conductance steps than the number of histogram peaks. It means that only some of the particular atomic arrangements appear consecutively one after the other during breaking of nanowires forming particular history of breaking. This rises the following questions about possible correlation between different arrangements: whether the presence of one particular arrangement determines the appearance of a different one, that is correlated with the first one, or on the contrary, whether some arrangements never follow some of the previous arrangements. In order to answer these questions we suggest a new type of analysis, namely, a correlation histogram. In Fig. 3 we present plot that contains information about correlations between different conductance plateau values from Fig. 2b. At the diagonal, there is exactly the usual histogram presented in Fig. 2b indicated by corresponding letters, and out of the diagonal, there are peaks that provide informa- tion about correlation between two different values of conductance as indicated at the vertical and horizontal axes. For example, there is strong correlation peak for $a$ and $c$ conductance histogram peaks. On the other hand, there is lack of the correlation for the peaks $a$ and $b$. It means that both atomic arrangements do not appear in the same sequence of arrangements formed during a particular breaking procedure. Following this analysis we can find three typical scenarios of the nanowire breaking. The first one (i) is $f-c-a$, the second (ii) $g-e-b$, and the third (iii) $f-d-a$. We can see that the scenario (iii) is just a small modification of (i), where the arrangement $c$ is replaced by $d$. However, both arrangements $c$ and $d$ are not correlated which means that they do never appear together. It is interesting that sequence (ii) is independent of (i) and (iii) since it does not share any common arrangements.

This new type of analysis can provide new information on correlation between different atomic arrangements formed consecutively during the formation and breaking of contacts in the metallic nanowires, that allow for more detailed comparison with advanced theoretical simulation of the formation of atomic QPCs.

\section{Acknowledgments}

We thank A. Halbritter and J.M. van Ruitenbeek for helpful discussions. This work was supported in part by the Polish grants for science in years 2006-2008 and 2006-2009 as a research projects. J.M. gratefully acknowledges the hospitality of the Centre de Physique Theorique - CNRS — Luminy, Marseille, France.

\section{References}

[1] J.I. Pascual, J. Méndez, J. Gómez-Herrero, A.M. Baró, N. García, Vu Thien Binh, Phys. Rev. Lett. 71, 1852 (1993).

[2] J.M. Krans, C.J. Muller, I.K. Yanson, Th.C.M. Govaert, R. Hesper, J.M. van Ruitenbeek, Phys. Rev. B 48, 14721 (1993).

[3] K. Hansen, E. Laegsgaard, I. Stensgaard, F. Besenbacher, Phys. Rev. B 56, 2208 (1997).

[4] J.L. Costa-Krämer, N. García, P. García-Mochales, P.A. Serena, M.I. Marqués, A. Correia, Phys. Rev. B 55, 5416 (1997).

[5] E. Scheer, P. Konrad, C. Bacca, A. Mayer-Gindner, H. v. Löhneysen, M. Häfner, J.C. Cuevas, Phys. Rev. B 74, 205430 (2006).

[6] R. Hasunuma, T. Komeda, H. Tokumoto, Appl. Surf. Sci. 130-132, 84 (1998).

[7] B. Susła, M. Wawrzyniak, J. Barnaś, W. Nawrocki, Mater. Sci. Poland 25, 305 (2007).

[8] T. Iwanari, T. Sakata, Y. Miyatake, S. Kurokawa, A. Sakai, J. Appl. Phys. 102, 114312 (2007).

[9] M. Wawrzyniak, Metrology Measur. Syst. 13, 161 (2006). 